\title{
Static Einstein-Maxwell Black Holes with No Spatial Isometries in AdS Space
}

\author{
Carlos A. R. Herdeiro and Eugen Radu \\ Departamento de Física da Universidade de Aveiro and CIDMA, Campus de Santiago, 3810-183 Aveiro, Portugal
}

(Received 9 June 2016; published 22 November 2016)

\begin{abstract}
We explicitly construct static black hole solutions to the fully nonlinear, $D=4$, Einstein-Maxwellanti-de Sitter (AdS) equations that have no continuous spatial symmetries. These black holes have a smooth, topologically spherical horizon (section), but without isometries, and approach, asymptotically, global AdS spacetime. They are interpreted as bound states of a horizon with the Einstein-Maxwell-AdS solitons recently discovered, for appropriate boundary data. In sharp contrast to the uniqueness results for a Minkowski electrovacuum, the existence of these black holes shows that single, equilibrium, black hole solutions in an AdS electrovacuum admit an arbitrary multipole structure.
\end{abstract}

DOI: 10.1103/PhysRevLett.117.221102

Introduction.-In 1967, Israel established a remarkable and influential result in black hole (BH) physics: a static, vacuum, regular (on and outside the horizon) $\mathrm{BH}$ in general relativity (GR) is spherically symmetric [1]. A corollary, indeed an application of Birkhoff's theorem, implies that such spacetime is the Schwarzschild $\mathrm{BH}$ and, hence, described by a unique parameter, its Arnowitt-DeserMisner mass. This result, in clear contrast with the status quo in other field theories (say, electromagnetism), set the first cornerstone for the celebrated uniqueness theorems [2], establishing the extraordinary simplicity of $\mathrm{BHs}$ in vacuum GR.

Israel's result was swiftly generalized to an electrovacuum [3], establishing that a single, static, $\mathrm{BH}$ solution is spherically symmetric and described by only two parameters, its Arnowitt-Deser-Misner mass and electric charge (excluding magnetic charges). The purpose of this Letter is to establish that the addition of a negative cosmological constant to the electrovacuum model, hereafter dubbed AdS electrovacuum, allows a dramatic departure from Israel's theorem: staticity does not guarantee the existence of any continuous spatial symmetry for physically acceptable BHs.

We establish this result by explicitly constructing the first, fully nonlinear, codimension 3 , equilibrium, single $\mathrm{BH}$ solutions in GR. As examples, we exhibit a sample of exotic $\mathrm{BH}$ horizon geometries, deprived of isometries, albeit possessing discrete symmetries, illustrated by their isometric embeddings in Euclidean 3-space.

The role of gravitating solitons.-Gravitating solitons are stationary, everywhere regular spacetimes with localized energy, i.e., particlelike solutions of GR (or extensions thereof). Influential examples, focusing on trivial spacetime topologies, have been found, e.g., in Einstein-complexKlein-Gordon theory, dubbed boson stars [4-6], or in Einstein-Yang-Mills (EYM) theory [7]. When gravitating solitons exist in a given model, bound states of such solitons with an event horizon can typically be constructed (see, e.g., Ref. [8]), leading to more complicated BHs, often called hairy [9]. For instance, placing a horizon inside the two above examples of gravitating solitons, leads, respectively, to Kerr BHs with scalar hair [11,12] and "colored" BHs [13-17].

This general principle indicates how departures from Israel's theorem can be constructed, using the fact that solitonic objects allow, typically, fewer symmetries. Indeed, explicit static gravitating solitons and $\mathrm{BHs}$ with only axial symmetry were constructed, e.g., in Refs. [18,19] within EYM theory. But something even more dramatic should be possible. A number of (nonlinear) field theories possess, on a Minkowski background, known static solitonic solutions without any continuous (spatial) symmetries (e.g., Refs. [20-23]), which must gravitate when coupled to GR. The addition of a horizon, therefore, will likely yield static BHs without any continuous (spatial) symmetries. Up to now, however, this maximal departure from Israel's theorem found no explicitly constructed realization; see Refs. [24,25] for partial results in this direction.

Recently, a new candidate model for this construction was unveiled: AdS electrovacuum. Classical results in GR established the inexistence of gravitating solitons in vacuum [26,27], electrovacuum [28], or AdS vacuum [29]. Remarkably, in AdS electrovacuum, and despite apparent obstructions [30], such solitons exist naturally. They were anticipated and constructed linearly in Ref. [31] by simple considerations of electrostatics in global AdS; fully noninearly examples were presented in Refs. [32] and [33]. In a nutshell, we note the following. (i) The boxlike structure of AdS allows the existence of electric (or magnetic) multipoles, as test fields, which are everywhere regular. They are defined by their multipole structure at the AdS boundary. (ii) Their backreaction yields Einstein-Maxwell-AdS solitons, which inherit the spatial symmetries of the boundary data. (iii) Introducing a horizon yields a static $\mathrm{BH}$ without continuous spatial symmetries, for appropriate boundary multipoles. A static, axially symmetric $\mathrm{BH}$ within a dipole soliton was constructed in Ref. [32]. Here, we construct static 
BHs without any spatial isometry, which, as we shall see, require solitons with higher multipoles.

Smooth electric multipoles on AdS electrovacuum.Einstein-Maxwell theory with a negative cosmological constant is described by the action

$$
\mathcal{S}=\frac{1}{16 \pi G} \int d^{4} x \sqrt{-g}\left\{R-2 \Lambda-F_{\mu \nu} F^{\mu \nu}\right\} .
$$

$F=d A$ is the $U(1)$ field strength and $\Lambda \equiv-3 / L^{2}<0$ is the cosmological constant, where $L$ is the AdS "radius." Varying the action, one obtains the Einstein-Maxwell equations, $G_{\mu \nu}+\Lambda g_{\mu \nu}=2 T_{\mu \nu}, \quad d \star F=0$, where the electromagnetic energy-momentum tensor is $T_{\mu \nu}=$ $F_{\mu \alpha} F_{\nu \beta} g^{\alpha \beta}-g_{\mu \nu} F^{2} / 4$. The maximally symmetric solution of this theory is AdS, with $F=0$, which in global coordinates reads

$$
d s^{2}=-N(r) d t^{2}+\frac{d r^{2}}{N(r)}+r^{2}\left(d \theta^{2}+\sin ^{2} \theta d \varphi^{2}\right),
$$

where $N(r)=1+r^{2} / L^{2}$. AdS electrostatics in global coordinates, for test fields, exhibits an important difference with respect to its Minkowski counterpart: there are everywhere regular solutions for all multipoles (except the monopole), which decay as $1 / r$, asymptotically [31]. A similar statement applies to magnetostatics [33]. In these previous studies, only the axisymmetric multipoles were considered. Here we consider the most general electrostatic potential, $A=V(r, \theta, \varphi) d t$, with

$$
V(r, \theta, \phi)=\sum_{\ell \geq 1} \sum_{m=-\ell}^{m=\ell} c_{\ell m} R_{\ell}(r) Y_{\ell m}(\theta, \varphi),
$$

where $c_{\ell m}$ are arbitrary constants and $Y_{\ell m}(\theta, \varphi)$ are the real spherical harmonics [34], normalized such that $\int d \Omega Y_{\ell m} Y_{\ell^{\prime} m^{\prime}}=\delta_{\ell \ell^{\prime}} \delta_{m m^{\prime}}$. Because of the AdS background symmetries, the radial equation is $m$ independent, $(d / d r)$ $\left\{r^{2}\left[d R_{\ell}(r) / d r\right]\right\}=[1 / N(r)] \ell(\ell+1) R_{\ell}(r)$. For $\ell \geq 1$ this equation possesses a solution which is regular everywhere (in particular at $r=0$ ), which can be written in terms of hypergeometric functions [31]:

$$
R_{\ell}(r)=\frac{\Gamma\left(\frac{1+\ell}{2}\right) \Gamma\left(\frac{3+\ell}{2}\right)}{\sqrt{\pi} \Gamma\left(\frac{3}{2}+\ell\right)} \frac{r^{\ell}}{L^{\ell}}{ }^{2} F_{1}\left(\frac{1+\ell}{2}, \frac{\ell}{2}, \frac{3}{2}+\ell,-\frac{r^{2}}{L^{2}}\right),
$$

where the normalization guarantees that $R_{\ell}(r) \rightarrow 1$ asymptotically.

The energy density of the solutions $\rho=-T_{t}^{t}$ is finite everywhere and strongly localized in a finite region of space, depending on both $\theta$ and $\varphi . \rho$ is nonzero at $\theta=0$; at $r=0$ it vanishes unless $\ell=1$. At infinity, $\rho$ decays as $1 / r^{4}$, such that the total energy of these solutions, $E=$ $-\int \sqrt{-g} T_{t}^{t} d^{3} x$, is finite. With the chosen normalization $E_{\ell}=L \Gamma(1+\ell / 1+\ell) \Gamma(3+\ell / 2) /\{\Gamma[1+(\ell / 2)] \Gamma(\ell / 2)\}$ [35].

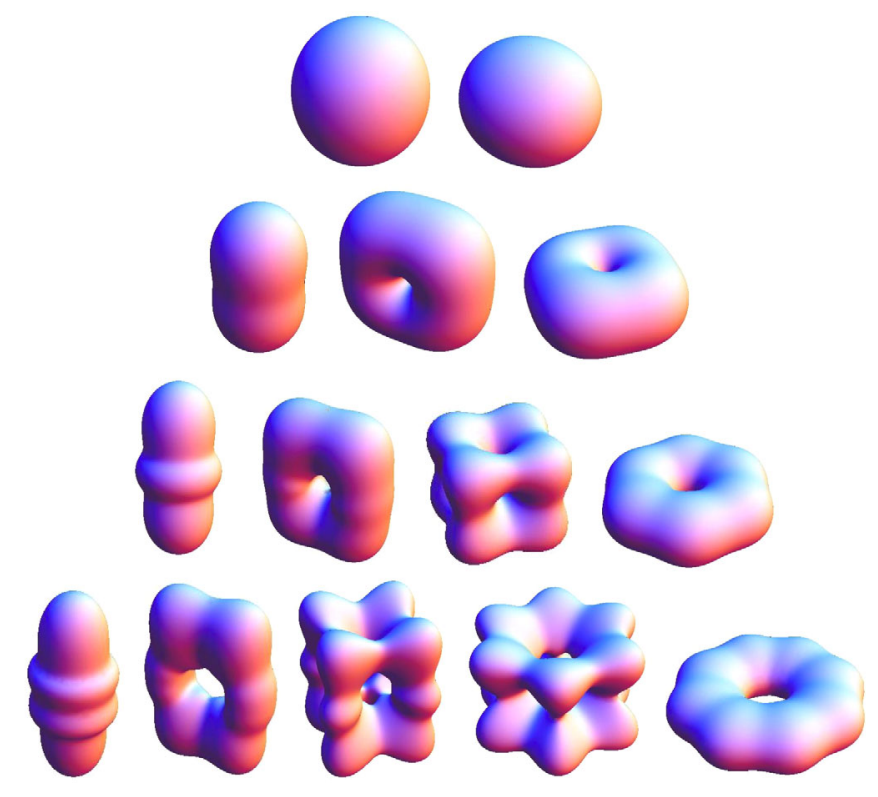

FIG. 1. Examples of surfaces of constant energy density for the Maxwell-AdS regular electric multipoles with (from left to right) $(\ell, m)=\{(1,0),(1,1)\} \quad$ (top row); $(\ell, m)=\{(2,0),(2,1)$, $(2,2)\} \quad($ second row); $(\ell, m)=\{(3,0),(3,1),(3,2),(3,3)\}$ (third row); $(\ell, m)=\{(4,0),(4,1),(4,2),(4,3),(4,4)\}$ (fourth row). Here we are defining standard Cartesian coordinates from the global AdS coordinates, using the standard formulas. All plots in this work use units with $L=G=1$.

These static regular electric multipoles on a fixed AdS background satisfy the virial identity

$$
\int_{0}^{\infty} r^{2} d r \int_{0}^{\pi} \sin \theta\left[V_{, r}^{2}+\frac{1-\frac{r^{2}}{L^{2}}}{N^{2}(r) r^{2}}\left(V_{, \theta}^{2}+\frac{V_{, \varphi}^{2}}{\sin ^{2} \theta}\right)\right]=0 .
$$

In the $L \rightarrow \infty$ limit (Minkowski), all terms in the integrand are positive definite and no nontrivial configurations can exist. This identity clarifies that (i) the AdS geometry supplies the attractive force needed to balance the repulsive gauge interactions, and (ii) the configurations are supported by the nontrivial angular dependence of $V$; i.e., they must possess a multipolar structure.

In Fig. 1 we exhibit surfaces of constant energy density for a sample of these solutions, with $\ell=1,2,3,4$ and $m \geq 0$ (the case $m<0$ follows directly). For $m \neq 0$, these surfaces possess solely discrete symmetries. The exception to this pattern occurs for $\ell=1$, wherein the $m=1$ and $m=0$ multipoles are related by a rotation (as indeed are the $\ell=2, m=1$, and $m=2$ multipoles). Thus, obtaining static BHs with no spatial isometries requires taking $\ell \geq 2$.

Similar solutions to the ones just described are found when taking instead a Schwarzschild-AdS (SAdS) BH backgound, with a line element still given by Eq. (2), where now $N(r)=\left(1-r_{H} / r\right)\left[1+\left(r^{2}+r r_{H}+r_{H}^{2}\right) / L^{2}\right]$, and $r_{H}>0$ is the event horizon radius. The corresponding radial equation cannot, however, be solved in closed form 
any longer (except for $\ell=0$ ). But it is straightforward to obtain a numerical solution, for any $\ell \geq 1$ [36]. The radial function vanishes on the horizon, in the neighborhood of which it can be written as a power series in $\left(r-r_{H}\right)$. Solutions are regular everywhere, on and outside the horizon, showing that these regular electric multipoles can be superimposed on the SAdS BH.

The nonlinear setup.-Fully nonlinear AdS-electrovacuum solitons and BHs are obtained from the backreaction of the above solutions. We shall tackle the fully nonlinear Einstein-Maxwell-AdS equations numerically, employing the Einstein-De Turck (EDT) method [37,38]. This approach to the numerical treatment of stationary problems in GR does not require fixing, a priori, a metric gauge, yielding, nevertheless, elliptic equations (see, e.g., Refs. [39,40] for reviews). The EDT equations are

$$
R_{\mu \nu}-\nabla_{(\mu} \xi_{\nu)}=\Lambda g_{\mu \nu}+2\left(T_{\mu \nu}-\frac{1}{2} T g_{\mu \nu}\right) .
$$

Here, $\xi^{\mu}$ is a vector defined as $\xi^{\mu} \equiv g^{\nu \rho}\left(\Gamma_{\nu \rho}^{\mu}-\bar{\Gamma}_{\nu \rho}^{\mu}\right)$, where $\Gamma_{\nu \rho}^{\mu}\left(\bar{\Gamma}_{\nu \rho}^{\mu}\right)$ is the Levi-Civita connection associated to the spacetime metric $g$ that one wants to determine (a reference metric $\bar{g}$ that is introduced). Solutions to Eq. (4) solve the Einstein equations if and only if $\xi^{\mu} \equiv 0$ everywhere on the manifold.

To solve Eq. (4), together with the Maxwell equations, we use an ansatz with seven unknown metric functions, $F_{1}$, $F_{2}, F_{3}, F_{0}, S_{1}, S_{2}, S_{3}$ and an electrostatic potential $V$ :

$$
\begin{aligned}
d s^{2}= & F_{1}(r, \theta, \varphi) \frac{d r^{2}}{N(r)}+F_{2}(r, \theta, \varphi)\left[r d \theta+S_{1}(r, \theta, \varphi) d r\right]^{2} \\
& +F_{3}(r, \theta, \varphi)\left[r \sin \theta d \varphi+S_{2}(r, \theta, \varphi) d r\right. \\
& \left.+S_{3}(r, \theta, \varphi) r d \theta\right]^{2}-F_{0}(r, \theta, \varphi) N(r) d t^{2}, \quad \text { and } \\
A= & V(r, \theta, \varphi) d t
\end{aligned}
$$

where $N(r)=\left[1-\left(r_{H} / r\right)\right]\left[1+\left(r^{2}+r r_{H}+r_{H}^{2} / L^{2}\right)-\left(q^{2} /\right.\right.$ $\left.r r_{H}\right)$ ] is a background function, with $r_{H}>0$ the event horizon radius and $q$ another input constant. Then the problem reduces to solving a set of eight PDEs with suitable boundary conditions (BCs). The BCs are found by constructing an approximate form of the solutions on the boundary of the domain of integration, compatible with the requirement $\xi^{\mu}=0$, plus regularity and $\mathrm{AdS}$ asymptotics. In particular, the first requirement should imply $\xi^{\mu} \equiv 0$ everywhere, a condition which is verified from the numerical output.

We have focused our study on $m>0$ solutions with a reflection symmetry along the equatorial plane $(\theta=\pi / 2)$ and two $\mathbb{Z}_{2}$ symmetries with respect to the $\varphi$ coordinate. The domain of integration for the $(\theta, \varphi)$ coordinates is then $[0, \pi / 2] \times[0, \pi / 2]$. Explicitly, we impose the following BCs at infinity (see also [41]) $F_{0}=F_{1}=F_{2}=F_{3}=1$, $S_{1}=S_{2}=S_{3}=0, V=c_{e} Y_{\ell m}(\theta, \varphi)$, which defines the
Maxwell boundary data to be a single harmonic $(\ell, m)$ with strength $c_{e}$. The $\mathrm{BCs}$ at $\theta=0$ are $\partial_{\theta} F_{0}=\partial_{\theta} F_{1}=$ $\partial_{\theta} F_{2}=\partial_{\theta} F_{3}=0, S_{1}=S_{2}=\partial_{\theta} S_{3}=0, V=0$. At $\theta=$ $\pi / 2$ we impose $\partial_{\theta} F_{0}=\partial_{\theta} F_{1}=\partial_{\theta} F_{2}=\partial_{\theta} F_{3}=0$, $S_{1}=\partial_{\theta} S_{2}=S_{3}=0$, together with $V=0$, except if $\ell+m$ is an even number, in which case we impose $\partial_{\theta} V=0$. The BCs at $\varphi=0$ are $\partial_{\varphi} F_{1}=\partial_{\varphi} F_{2}=\partial_{\varphi} F_{3}=$ $\partial_{\varphi} F_{0}=0, \partial_{\varphi} S_{1}=S_{2}=S_{3}=0, \partial_{\varphi} V=0$. At $\varphi=\pi / 2$ we impose $\partial_{\varphi} F_{1}=\partial_{\varphi} F_{2}=\partial_{\varphi} F_{3}=\partial_{\varphi} F_{0}=0, \partial_{\varphi} S_{1}=S_{2}=$ $S_{3}=0$ together with $V=0$ for odd $m$, or $\partial_{\varphi} V=0$ for even $m$. Solitonic solutions have $r_{H}=0=q$ and the range of the radial coordinate is $0 \leq r<\infty$. At $r=0$ we impose $\partial_{r} F_{1}=\partial_{r} F_{2}=\partial_{r} F_{3}=\partial_{r} F_{0}=\partial_{r} S_{1}=\partial_{r} S_{2}=\partial_{r} S_{3}=0$, $V=0$. The BHs have a horizon located at $r=r_{H}>0$. To deal with the BCs there, it proves useful to introduce a new (compact) radial coordinate $x$, as $r \equiv\left[r_{H} / 1-(x / 2 L)^{2}\right]$, such that $0 \leq x<2 L$ and in terms of which the horizon is located at $x=0$. This yields the following BCs at the horizon: $\partial_{x} F_{1}=\partial_{x} F_{2}=\partial_{x} F_{3}=\partial_{x} F_{0}=0, S_{1}=S_{2}=$ $\partial_{x} S_{3}=0, V=0$.

Numerical procedure.-We have successfully obtained numerical solutions for both BHs and solitons in AdS electrovacuum, fixing the gauge field boundary data to be a single $Y_{\ell m}$ harmonic, and scanning through a variety of $\ell$, $m$ values. The numerical procedure we have used is a modified version of the approach previously employed in the study of axially symmetric configurations of the same model [33]. The field equations are first discretized on a $(r, \theta, \varphi)$ grid with $N_{r} \times N_{\theta} \times N_{\varphi}$ points. The grid spacing in the $r$ direction is nonuniform, while the values of the grid points in the angular directions are uniform. Typical grids have sizes $\sim 100 \times 30 \times 30$. The resulting system is solved iteratively until convergence is achieved. Computations are performed by adapting a finite difference code described in Ref. [42] based on the iterative Newton-Raphson method. For the solutions herein, the typical numerical error is estimated to be $\lesssim 10^{-3}$.

In practice, the $\mathrm{BH}$ solutions are found starting with SAdS BHs and slowly increasing the parameter $c_{e}$ in the BCs at infinity. In a second step, the parameters $\left(r_{H}, q\right)$ in Eq. (5) are also varied.

Horizon geometry.-The most unusual property of the generic $\mathrm{BH}$ solutions is that their horizons do not possess a rotational symmetry, despite being topologically a 2-sphere. To establish this result, we consider the induced metric at the horizon, which reads, from Eq. (5),

$$
d \sigma^{2}=r_{H}^{2}\left[F_{2} d \theta^{2}+F_{3}\left(\sin \theta d \varphi+S_{3} d \theta\right)^{2}\right],
$$

where $F_{2}, F_{3}, S_{3}$ are now only functions of $\theta, \varphi$. To visualize this geometry, we consider its isometric embedding in a flat three-dimensional space, with $d \sigma^{2}=$ $d X^{2}+d Y^{2}+d Z^{2}$, the embedding functions, $X(\theta, \varphi)$, $Y(\theta, \varphi), Z(\theta, \varphi)$, being found by integrating a system of nonlinear PDEs. In Fig. 2 we exhibit 3D isometric 

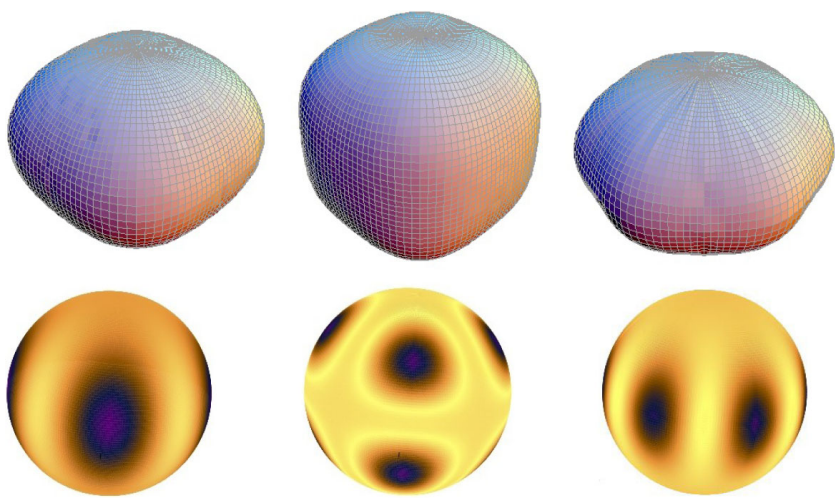

FIG. 2. Examples of isometric embeddings for the horizon of AdS-electrovacuum BHs (top), together with their horizon Ricci scalar (bottom). The boundary data are given by the harmonics with $(\ell, m)$ equal to $(2,2)$ (left), $(3,2)$ (middle), and $(3,3)$ (right).

embeddings for a set of nonaxisymmetric BHs. For instance, for $(\ell, m)=(3,2)$ boundary data, one obtains a cubiclike horizon. Comparing with Fig. 1 one observes that the horizon loosely adapts to the corresponding constant energy surface, except that it is topologically simply connected. Also, the horizon scalar curvature is everywhere finite, although it can take large values [43]. As shown in Fig. 3, the horizon deformation increases with $c_{e}$; these global isometric embeddings, however, can only be obtained up to some threshold value of $c_{e}$, beyond which well-known obstructions arise (see, e.g., Refs. [44,45]).

Global charges and thermodynamics. - These configurations carry a nonzero electric charge density; their total electric charge, however, vanishes. As such, the only global charge of the solutions is their mass $M$. Its expression, computed by employing either the prescriptions in Ref. [46] or the one in Ref. [47], is

$$
M=M^{(b)}-\frac{3 L}{16 \pi G} \int_{0}^{2 \pi} d \varphi \int_{0}^{\pi} d \theta \sin \theta f_{03}(\theta, \varphi),
$$
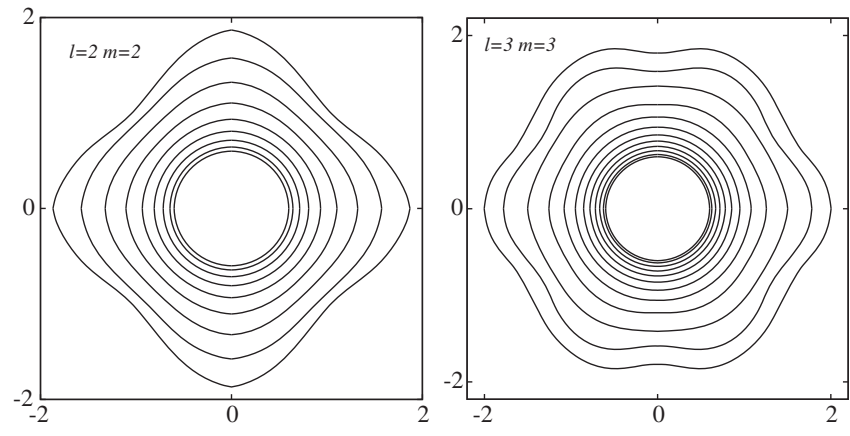

FIG. 3. Equatorial slices for isometric embeddings of the horizons of AdS-electrovacuum BHs with different boundary data. The BHs have the same temperature and increasing values of the parameter $c_{e}$, starting with $c_{e}=0$ (center). where $\quad M^{(b)}=\left(r_{H} / 2 G\right)\left[1+\left(r_{H}^{2} / L^{2}\right)+\left(q^{2} / r_{H}^{2}\right)\right] \quad$ is a contribution from the background metric and $f_{03}(\theta, \varphi)$ is a function which enters the far field asymptotics, with $F_{0}=1+f_{03} / r^{3}+\cdots$. In Fig. 4 we exhibit the total mass for BH solutions with different $(\ell, m)$ values of boundary data, for a fixed temperature and varying $c_{e}$. The pattern is universal: the mass increases with $c_{e}$ and also (for the same $m$ ) with the multipole number $\ell$.

Of interest are also the horizon area and Hawking temperature of the $\mathrm{BHs}$,

$A_{H}=\int_{0}^{2 \pi} d \varphi \int_{0}^{\pi} d \theta \sqrt{g_{\sigma}}, \quad T_{H}=\frac{\left(1+3 r_{H}^{2} / L^{2}-q^{2}\right)}{4 \pi r_{H}}$,

where $\sqrt{g_{\sigma}}=r_{H}^{2} \sin \theta \sqrt{F_{2} F_{3}}$. In the absence of a net electric charge, the thermodynamics has similarities to that of SAdS BHs. As shown in Fig. 4 (inset), there are two branches of BHs, existing above a minimal temperature $T_{H}^{(\min )}>0$, where $T_{H}^{(\min )}$ decreases with $c_{e}$. For lower branch solutions, the $\mathrm{BH}$ size decreases with $T_{H}$, while for upper branch BHs, the horizon area increases with the temperature, with no upper bound on $A_{H}$.

These $\mathrm{BH}$ solutions possess a nontrivial zero horizon size limit $r_{H} \rightarrow 0$, corresponding to AdS-electrovacuum solitons with no isometries. The $\left(M, c_{e}\right)$ diagram of the solitons is similar to that exhibited for BHs in Fig. 4.

Finally, let us mention two generalizations. (i) These BHs can be endowed with a net electric charge by turning on an additional $\ell=0$ mode, in the boundary condition for $V$ at infinity. Such solutions, however, do not possess a solitonic limit and can be thought of as describing the (nonlinear) superposition of a Reissner-Nordström (rather than Schwarzschild) BH with AdS-electrovacuum solitons. (ii) The configurations described herein will possess a magnetic dualized version, which can be straighforwardly constructed.

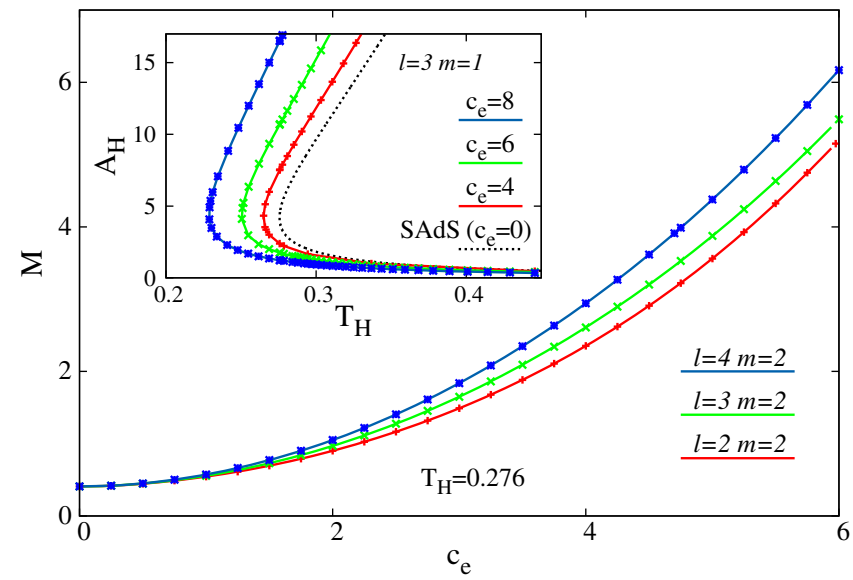

FIG. 4. Mass vs $c_{e}$ for families of BHs with different boundary data and the same temperature. (Inset) Horizon area vs temperature for $(3,1) \mathrm{BHs}$ with different values of $c_{e}$. 
Remarks.-Static (single) BHs in electrovacuum can only have an electric monopole, and they are necessarily spherically symmetric. In sharp contrast, static BHs in AdS electrovacuum can have an arbitrary electric multipole structure; by turning on appropriate multipoles, we have presented explicit examples of static BHs with no continuous (spatial) symmetries.

The BHs presented here still exhibit discrete symmetries. Is it possible, with appropriate boundary data, to obtain $\mathrm{BH}$ horizons, in AdS electrovacuum, isometric to any topologically spherical 2-manifold? If not, what 2-geometries are allowed? Whatever the correct answer is, the results reported herein show (yet) another example of how conceptually different AdS gravity is from its Minkowski space counterpart.

The authors acknowledge funding from the FCT-IF programme. This work was partially supported by the H2020-MSCA-RISE-2015 Grant No. StronGrHEP-690 904, and by the CIDMA Project No. UID/MAT/04106/ 2013. Computations were performed at the Blafis cluster, in Aveiro University.

[1] W. Israel, Phys. Rev. 164, 1776 (1967).

[2] P. T. Chrusciel,J. Costa and M. Heusler, Living Rev. Relativ. 15, 7 (2012).

[3] W. Israel, Commun. Math. Phys. 8, 245 (1968).

[4] D. J. Kaup, Phys. Rev. 172, 1331 (1968).

[5] R. Ruffini and S. Bonazzola, Phys. Rev. 187, 1767 (1969).

[6] F. Schunck and E. Mielke, Classical Quantum Gravity 20, R301 (2003).

[7] R. Bartnik and J. McKinnon, Phys. Rev. Lett. 61, 141 (1988).

[8] D. Kastor and J. H. Traschen, Phys. Rev. D 46, 5399 (1992).

[9] This is, however, no golden rule. A famous example is spherically symmetric boson stars, which admit no black hole generalization [10]. This example emphasizes that symmetry compatibility between the soliton and horizon is a nontrivial necessary condition to place the latter inside the former.

[10] I. Pena and D. Sudarsky, Classical Quantum Gravity 14, 3131 (1997).

[11] C. A. R. Herdeiro and E. Radu, Phys. Rev. Lett. 112, 221101 (2014).

[12] C. Herdeiro and E. Radu, Classical Quantum Gravity 32, 144001 (2015).

[13] M. S. Volkov and D. V. Galtsov, Pis'ma Zh. Eksp. Teor. Fiz. 50, 312 (1989) [JETP Lett. 50, 346 (1989)].

[14] M. S. Volkov and D. V. Galtsov, Yad. Fiz. 51, 1171 (1990) [Sov. J. Nucl. Phys. 51, 747 (1990)].

[15] H. P. Künzle and A. K. M. Masood-ul-Alam, J. Math. Phys. (N.Y.) 31, 928 (1990).

[16] P. Bizon, Phys. Rev. Lett. 64, 2844 (1990).

[17] M. S. Volkov and D. V. Gal'tsov, Phys. Rep. 319, 1 (1999).

[18] B. Kleihaus and J. Kunz, Phys. Rev. Lett. 78, 2527 (1997).
[19] B. Kleihaus and J. Kunz, Phys. Rev. Lett. 79, 1595 (1997).

[20] C. J. Houghton and P. M. Sutcliffe, Commun. Math. Phys. 180, 343 (1996).

[21] L. D. Faddeev and A. J. Niemi, Nature (London) 387, 58 (1997).

[22] R. A. Battye and P. M. Sutcliffe, Phys. Rev. Lett. 79, 363 (1997).

[23] R. A. Battye and P. Sutcliffe, Proc. R. Soc. A 455, 4305 (1999).

[24] S. A. Ridgway and E. J. Weinberg, Phys. Rev. D 52, 3440 (1995).

[25] T. Ioannidou, B. Kleihaus and J. Kunz, Phys. Lett. B 635, 161 (2006).

[26] A. Einstein and W. Pauli, Ann. Math. 44, 131 (1943).

[27] A. Lichnerowicz, C.R. Hebd. Seances Acad. Sci. 222, 432 (1946).

[28] M. Heusler, Helv. Phys. Acta 69, 501 (1996).

[29] W. Boucher, G. W. Gibbons, and G. T. Horowitz, Phys. Rev. D 30, 2447 (1984).

[30] T. Shiromizu, S. Ohashi, and R. Suzuki, Phys. Rev. D 86, 064041 (2012).

[31] C. Herdeiro and E. Radu, Phys. Lett. B 749, 393 (2015).

[32] M. S. Costa, L. Greenspan, M. Oliveira, J. Penedones, and J.E. Santos, Classical Quantum Gravity 33, 115011 (2016).

[33] C. Herdeiro and E. Radu, Phys. Lett. B 757, 268 (2016).

[34] E. T. Whittaker and G. N. Watson, A Course of Modern Analysis, Cambridge Mathematical Library (Cambridge University Press, Cambridge, England, 1996).

[35] This value differs from the one in Ref. [31], due to a different normalization of the spherical harmonics.

[36] O. Kichakova, J. Kunz, E. Radu, and Y. Shnir, Phys. Rev. D 93, 044037 (2016).

[37] M. Headrick S. Kitchen, and T. Wiseman, Classical Quantum Gravity 27, 035002 (2010).

[38] A. Adam, S. Kitchen and T. Wiseman, Classical Quantum Gravity 29, 165002 (2012).

[39] T. Wiseman, arXiv:1107.5513.

[40] O. J. C. Dias, J. E. Santos and B. Way, Classical Quantum Gravity 33, 133001 (2016).

[41] See Supplemental Material at http://link.aps.org/ supplemental/10.1103/PhysRevLett.117.221102 for further details on the asymptotic expansion of the metric functions and electric potential.

[42] W. Schonauer and R. Weiss, J. Comput. Appl. Math. 27, 279 (1989).

[43] As a further test of our solutions, we have verified the Gauss-Bonnet theorem holds at the level of the numerical accuracy, by integrating the horizon Ricci scalar over the horizon.

[44] L. Smarr, Phys. Rev. D 7, 289 (1973).

[45] G. W. Gibbons, C. A. R. Herdeiro, and C. Rebelo, Phys. Rev. D 80, 044014 (2009).

[46] V. Balasubramanian and P. Kraus, Commun. Math. Phys. 208, 413 (1999).

[47] A. Ashtekar and S. Das, Classical Quantum Gravity 17, L17 (2000). 